\title{
ERRATUM
}

\section{A tutorial survey of architectures, algorithms, and applications for deep learning - ERRATUM}

\section{DENG}

doi:10.1017/ATSIP.2013.99. Published online by Cambridge University Press 22 January 2014.

In the above publication by Deng (2014), figure 2 has been mistakenly duplicated replacing figure 3 . The publisher apologises for this error and the correct figure 3 is shown below.

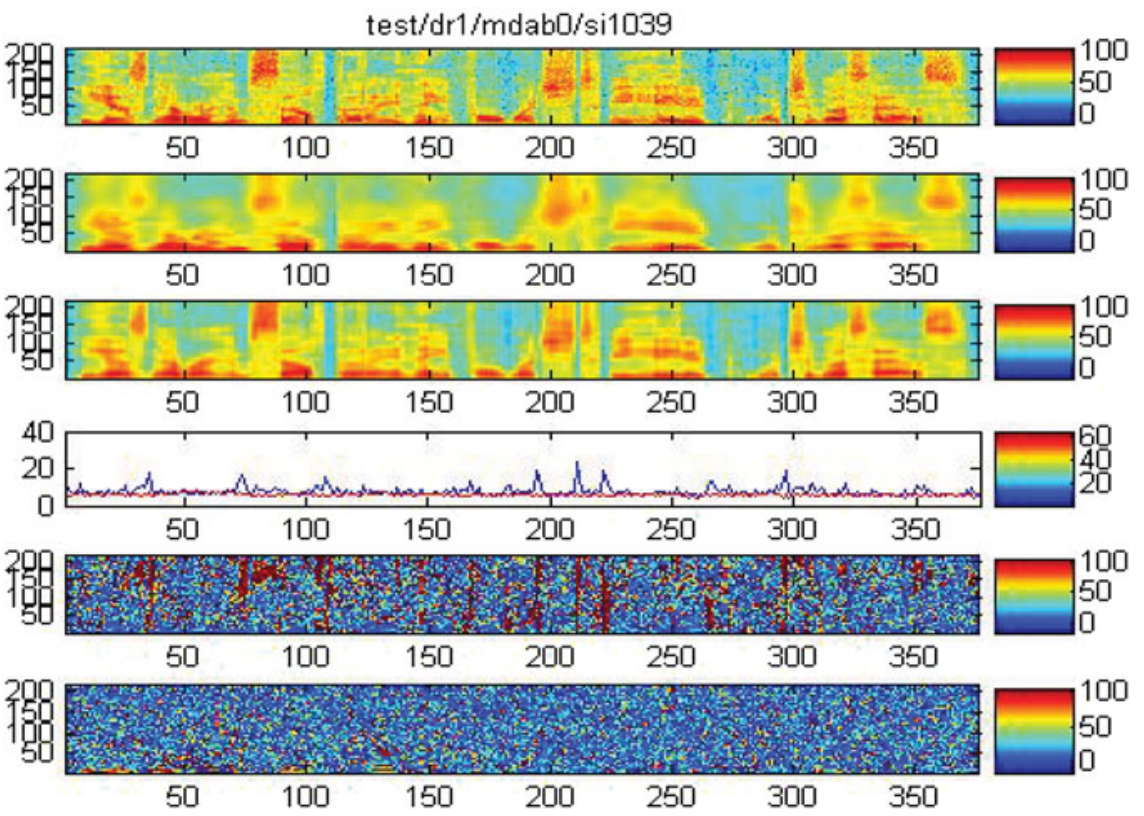

Fig. 3. Top to bottom: Original spectrogram from the test set; reconstruction from the 312-bit VQ coder; reconstruction from the 312-bit auto-encoder; coding errors as a function of time for the VQ coder (blue) and auto-encoder (red); spectrogram of the VQ coder residual; spectrogram of the deep autoencoder's residual.

\section{REFERENCE}

[1] Deng. L (2014). A tutorial survey of architectures, algorithms, and applications for deep Learning. APSIPA Transactions on Signal and Information Processing, 3, e2. doi:10.1017/ATSIP.2013.9. 